\title{
On fully superconducting rectifiers and fluxpumps. A review. Part 2: Commutation modes, characteristics and switches
}

\author{
L.J.M. van de Klundert and H.H.J. ten Kate
}

\begin{tabular}{|c|c|c|c|}
\hline \multicolumn{2}{|c|}{ Nomenclature } & \multirow{2}{*}{$\begin{array}{l}P_{\mathrm{L}}(i) \\
P_{\text {Loss }}(i)\end{array}$} & \multirow{2}{*}{$\begin{array}{l}\text { power to the load coil, } W \\
\text { power loss in flux pump, } W\end{array}$} \\
\hline$B$ & magnetic field, $\mathrm{T}$ & & \\
\hline$E_{\mathrm{L}}(i)$ & stored energy in load coil, $\mathrm{J}$ & $Q_{\text {in }}$ & input heat, $\mathbf{J}$ \\
\hline$E_{\text {loss }}(i)$ & energy loss, $\mathbf{J}$ & $Q_{\text {out }}$ & output heat, $\mathbf{J}$ \\
\hline$f$ & frequency, $s^{-1}$ & $R$ & resistance of open switch in rectifiers; effec- \\
\hline$\mu_{0} H_{0_{2}}$ & second critical field, $\mathrm{T}$ & & tive resistance of the normal spot in dynamos, $\Omega$ \\
\hline iI & normalized current, $I_{\mathrm{L}} / I_{\max }$, & $\Delta t_{\mathrm{i}}$ & time interval, $\mathrm{s}$ \\
\hline$\delta I$ & commutation step in primary current, A & $t$ & time, $s$ \\
\hline$\Delta I$ & current increase, $\mathrm{A}$ & $T$ & temperature, $\mathrm{K}$ \\
\hline$I$ & current, A & $T_{\mathrm{c}}$ & critical temperature, $\mathrm{K}$ \\
\hline$I_{\mathrm{L}}(t)$ & load current, A & $V$ & voltage, $\mathrm{m}^{3}$ \\
\hline$I_{\max }$ & maximum load current, $\mathrm{A}$ & $\bar{V}_{\mathbf{L}}$ & average voltage across the load coil, $\mathrm{V}$ \\
\hline$\hat{I}_{\mathrm{p}}$ & amplitude of primary current, $\mathrm{A}$ & $V_{\mathrm{R}}$ & voltage across a resistance $R, \mathrm{~V}$ \\
\hline$I_{\mathrm{s}}$ & secondary current, $\mathrm{A}$ & $\alpha, \beta$ & geometrical factors of dynamos \\
\hline$J_{\mathrm{c}}$ & critical current density, $\mathrm{Am}^{-2}$ & $\delta$ & $\begin{array}{l}\text { effective fraction of half a period used for } \\
\text { pumping }\end{array}$ \\
\hline$k$ & coupling coefficient of the transformer & & pumping \\
\hline$k_{\mathrm{B}}$ & Boltzmanns constant, $\mathrm{JK}^{-1}$ & $\eta_{\mathrm{E}}(i)$ & energy efficiency, $\%$ \\
\hline$l$ & $\begin{array}{l}\text { selfinductance of the secondary branch in } \\
\text { rectifiers; effective selfinductance of a normal } \\
\text { spot in dynamos, } \mathrm{H}\end{array}$ & $\begin{array}{l}\eta_{\mathrm{p}}(i) \\
\mu_{0} \\
\nu\end{array}$ & $\begin{array}{l}\text { power efficiency, } \% \\
\text { permeability of vacuum, } \mathrm{kg} \mathrm{mC}^{-2} \\
\text { laser frequency, } \mathrm{s}^{-1}\end{array}$ \\
\hline$l_{\mathrm{p}}$ & $\begin{array}{l}\text { selfinductance of the primary transformer } \\
\text { coil, } H\end{array}$ & $\rho$ & resistivity, $\Omega \mathrm{m}$ \\
\hline$L$ & selfinductance of the load coil, $\mathrm{H}$ & $\rho_{\mathbf{n}}$ & resistivity of a superconductor at $T=T_{\mathrm{c}}, \Omega \mathrm{m}$ \\
\hline $\begin{array}{l}M \\
n\end{array}$ & $\begin{array}{l}\text { mutual inductance of total transformer, } \mathrm{H} \\
\text { integer }\end{array}$ & $\phi_{0}$ & $\begin{array}{l}\text { constant input flux; } B_{\text {external }} \text { spot area in } \\
\text { dynamos; } 2 \hat{I}_{\mathrm{p}} M \text { in rectifier } ; \mathrm{W}_{\mathrm{b}}\end{array}$ \\
\hline 8 & Phys & $\phi(t)$ & $\begin{array}{l}\text { time dependent input flux: } B_{\text {external }}(t) \text { spot } \\
\text { area in dynamos; } 2 \hat{I}_{\mathrm{p}}(t) M \text { in rectifiers, } \mathrm{W}_{\mathrm{b}}\end{array}$ \\
\hline
\end{tabular}


In the previous part ${ }^{156}$ of this work, the basic principles and designs of fluxpumping were treated. Three types have been distinguished, namely the fluxcompressors, the sc dynamos and the sc transformer rectifiers. The work is continued in this part with a further analysis of the most important type of flux pump eg the fullwave and half wave superconducting transformer rectifiers. They both consist of a superconducting transformer and two sc switches arranged as shown in Fig. 24A.

The different modes of operation, loss mechanisms, transformer and some typical results are discussed below. The rectifier switches are of crucial importance for the feasibility of high current, high power, rectifier flux pumps. The different possibilities such as thermally, magnetically or mechanically activated switches are to be evaluated. The comparable theoretical behaviour of both dynamos and rectifiers is presented. The readers attention is drawn to the coherence between part 1 and part 2 of this review.

\section{Design aspects of sc rectifiers}

The flux pump as expressed here is an inductively loaded superconducting rectifier. Its behaviour differs from the normal conducting ones owing to the periodic current increase in the load coil until a maximum is reached. This pump behaviour is achieved by driving the transformer with an ac current and appropriate control of both switches. The pump's performance is harmfully effected by several more or less important loss contributions. The following loss terms are potentially undesirable but partly inevitable: Resistive commutation of the secondary current from one loop to the other; ohmic losses in the open switch with normal resistance $R$ due to a leakage current; core losses in transformer and in the saturation reactors if used; ac losses in wires of transformer and switch coils, and in input and control leads; thermal losses in case of thermally activated switches; ohmic losses due to imperfect 'superconducting' joints in the high current secondary circuit; ohmic losses in the protection resistance connected across the load cell, if needed; any possible mechanical loss due to insufficient fixed leads or components or in the mechanical switches if used; and eddy current losses due to magnetic stray fields.

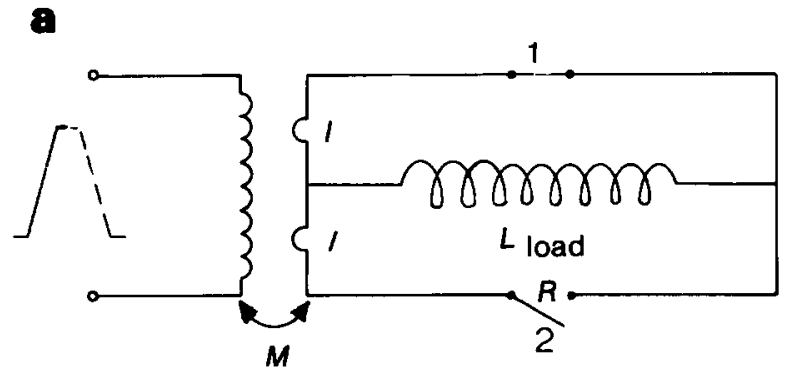

b

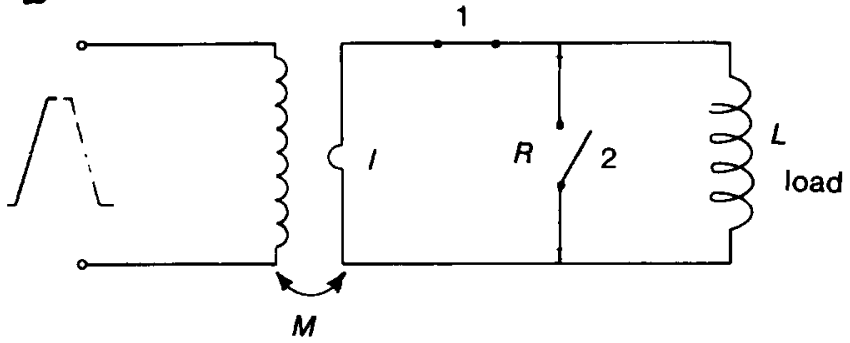

Fig. 24A a - Fullwave superconducting rectifies; $b$ - halfwave superconducting rectifier
For a high efficient flux pump these loss terms have to be eliminated or minimized. Only three authors report on the relative sizes of these loss contributions..$^{95,107,123}$ The losses in the magnetic or thermal switches are dominant (50-80\%); the transformer losses (15-35\%) to a lesser degree

The commutation losses can be substantially reduced or completely avoided as discussed below. Large load coils with a stored energy greater than about $25 \mathrm{~kJ}$ need protection against quenching in order to avoid damage to the coil and for safety of the whole system.

Some protection systems make use of an extra small ohmic shunt across the load coil. ${ }^{114,117,123}$ Such an ohmic loading of the flux pump influences the pump characteristics and causes extra losses so that it should be considered carefully.

Commutation losses occur because between the two half cycles at the maximum or minimum of the primary current while both switches are closed, the load current must be switched from one loop to the other in order to preserve the load current. If this is done simply by opening one switch, the energy in the loop proportional to $\tilde{I}_{\mathrm{L}}^{2}$ will be dissipated. For the half wave rectifier, in each pump cycle the stored energy is dissipated twice or $2 f$ times per second which gives a power loss $f l_{\mathrm{s}} I_{\mathrm{L}}^{2}$ dissipated in the resistive switch (see the following section on flux pump characterisitcs). $l_{\mathrm{s}}$ is the total self inductance of that branch of the circuit that contains the switch and the transformer secondary. For the full wave rectifier, twice as much stored energy is lost per second. The commutation losses appear because of the non-zero current through the switch during the switch process. This is called the resistive commutation or transfer mode.

A number of precautions can eliminate or minimize these losses:

One, a switch is not allowed to open until the current in the switch goes through zero as a result of the primary voltage. In this way the current is inductively driven out from one loop and switch. Because both loops are superconducting, flux conservation in combination with the transformer induction voltage takes care of a current decrease until it is zero in this loop. There is a similar increase in the other loop in which, in the following half cycle, the pump action takes place. This means that the secondary currents must be measured; when the current becomes zero, a control box must drive the switch to the resistive state. Switches may, however, have a finite recovery time and rise time so that the current through the switch will not be zero during the complete switching time.

Two, the use of precaution 1 and suitable saturation reactors with a nearly rectangular hysteresis loop (Fig. 25a) series connected with the switches, makes the secondary current in the switch a small constant value for a certain length of time, if the current has passed through zero. This time interval must be long enough in comparison with the switch rise time. A device working in this way has been built ${ }^{94,95,104}$ and is shown in Fig. 25b.

A disadvantage of this method is the need for a control device and an increased secondary selfinductance in series with the transformer secondary selfinductance $l$, so that the pump characteristics are disadvantageously effected.

Three, instead of minimizing the commutation losses with saturation reactors in the secondary circuit, it is also possible 

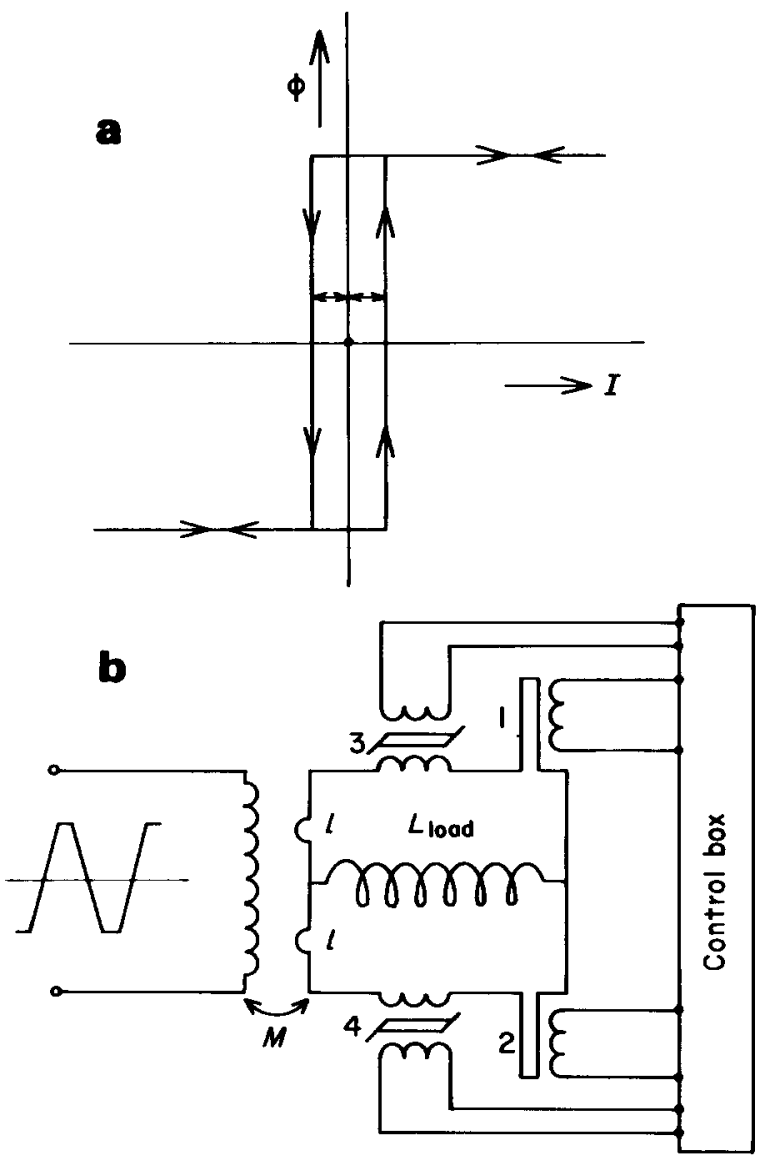

Fig. 25 a - Rectangular hysteresis loop of the saturation reactor; b - Complete full wave rectifier with saturation reactors $(3,4)$ and two magnetic switches $(1,2)$

to eliminate these losses simply by not changing the primary current during the switching, if the current secondary in the switch has to be zero. This can be done with a function generator which makes, for example, the primary current wave forms of Fig. 26a or b.

At the end of the pumping period, the primary current is constant for a moment $\Delta t_{1}$. The second switch closes so that both secondary loops become superconducting. A suitable step $\delta I$ in the primary current induces a circulating current in the short-circuited secondary of the transformer, which makes the current in the other switch zero. Then the primary current remains constant during a time interval $\Delta t_{2}$ and allows the 'current-zero' switch to be opened without any losses. The time in tervals $\Delta t_{1}$ and $\Delta t_{2}$ are made sufficiently large, to match the time constants of the switches. The secondary load current must be measured and fed back to the function generator which regulates $\delta I$.

Since $\delta I$ increases from zero at the beginning to a maximal $2 \hat{I}_{\mathrm{p}}$ at the end, the effective pumped flux becomes a function of time and decreases from $2 M \hat{I}_{\mathrm{p}}$ initially to zero when the maximum current is reached. The effect of a decreasing pump amplitude is compensated for by the profit of decreased losses and increased efficiency.

The pump characteristics with this perfect inductive commutation mode are changed for the better, which will become clear later.

The benefit of this commutation method is that principally every commutation loss is eliminated if the switch current is measured exactly. The control of such a device can easily be done with modern electronics. ${ }^{121,125,128}$ The points of time upon which both switches are driven are derived from the primary wave form by digital logic equipment.

The use of one or three phase (sinusoidal) power directly for the flux pump control seems very attractive but unfortunately makes the flux pump less efficient. It is clear from the above that sinusoidal wave forms are not the most suitable for driving a flux pump. The high frequency $(50-60 \mathrm{~Hz}) \mathrm{de}-$ creases the efficiency also, as will become clear.

Transformer. From the theory given later, it appears that for maximum power, current and efficiency the transformed flux $\phi=2 \hat{I_{\mathrm{p}}} M$ has to be large and the transformer secondary self inductance $l$ has to be small.

For the ideal full wave rectifier,

$$
I_{\max }=\frac{\phi}{2 l}=\frac{\hat{I}_{\mathrm{p}} M}{l}=I_{\mathrm{p}} k \frac{2 l_{\mathrm{p}}^{1 / 2}}{l}
$$

If the flux pump operates at maximum power $\left(I_{\mathrm{L}}=1 / 2 I_{\max }\right)$ and has a value for $l$ of about $10^{-8} \mathrm{H}$, a coupling coefficient $k$ of 0.5 , and operates with a $10 \mathrm{~A}$ primary current amplitude, the primary self inductance becomes minimal, $8 \times 10^{-4} x^{2} \mathrm{H}$ for $x \mathrm{kA}$. This means for $1 \mathrm{kA}$, it is $0.8 \mathrm{mH}$; for $10 \mathrm{kA}$, it is $80 \mathrm{mH}$ and for $100 \mathrm{kA}$ there is a primary selfinductance of $8 \mathrm{H}$.

It is absolutely essential to optimize the coupling coefficient and to make the secondary self inductance as small as possible.

From the load curve and (2.1), it becomes evident that for maximum current, there is a fundamental contradiction between short pumping time (high power) of load-current $(\sim L / l)$ and a large maximum current $\left(\sim l_{\mathrm{p}} / l\right)$. For large $k$ and large $l_{\mathrm{p}}$, a short, thick transformer form with many primary turns is preferable.

The primary selfinductance determines the primary rise time and also the requirement for a high voltage/low current
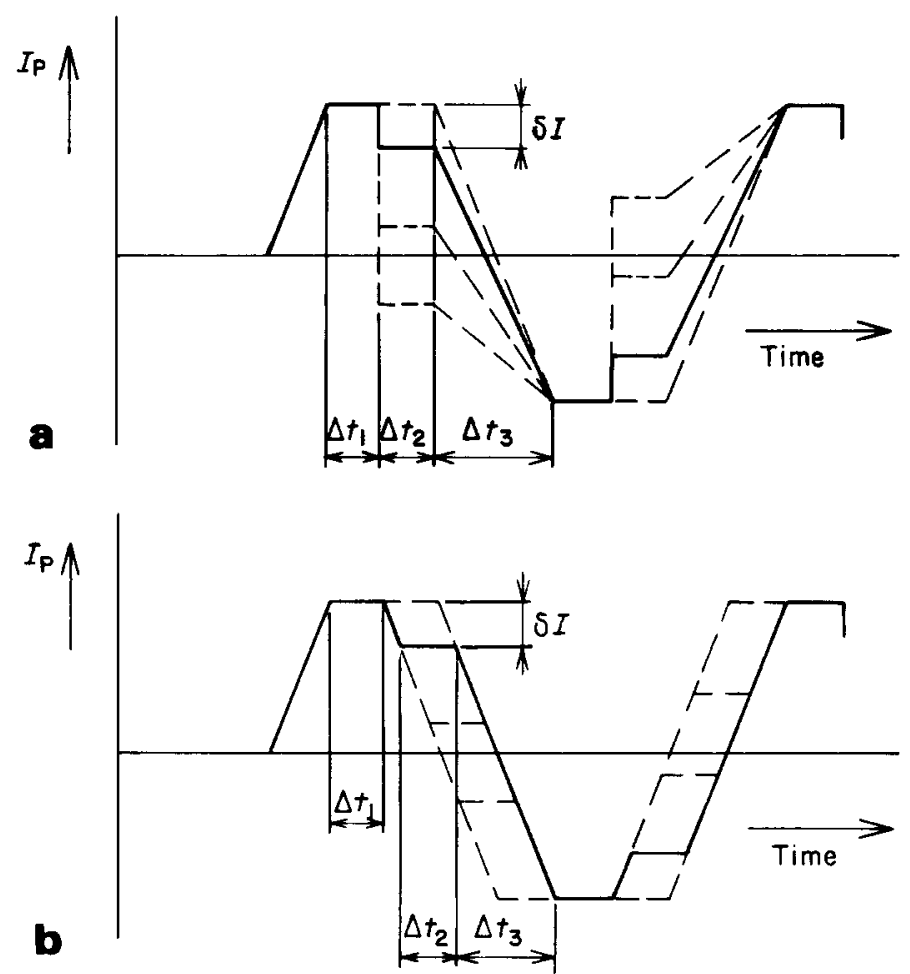

Fig. 26 Three possible primary current wave forms 
power supply (in references usually $10-20 \mathrm{~A} / 20-200 \mathrm{~V}$; $0.1-5 \mathrm{~Hz}$ ).

The primary rise time sets a limit to the frequency of operation. An iron or ferrite core increases the coupling coefficient, but makes the coupling non-linear and causes extra hysteretic core losses. For the centre-tapped transformers, the mutual coupling of the secondary windings has to be made negligibly small.

Improvements of the primary-secondary coupling however, also increase the secondary mutual coupling which again decreases the effective primary-secondary coupling.

Solenoid transformers are common. Cylindrical shields $\left(\mathrm{Nb}_{3} \mathrm{Sn}, \mathrm{Nb} \mathrm{Ti}\right)$ or iron return yokes prevent stray fields and also improve coupling.

Typically transformer turn ratios are in the range of 40:1 to $1000: 1$.

Few primary turns need large, unwanted primary currents; many turns make the transformer large, the fluxpump slow and high voltage supply necessary. For primary windings, $\mathrm{Nb}_{3} \mathrm{Sn}$, or $\mathrm{NbTi}$ multifilament superconductors in CuNi matrix with thicknesses in the range $100-300 \mu \mathrm{m}$ are common. At the secondary side, high current conductors are needed. Thick multifilament cables are possible, but give lower coupling coefficients than tape conductors. Many parallel wires reduce $l$ but have the disadvantage of counter currents, unequal current sharing, current instability and difficult joint techniques. The best results are reported with $\mathrm{Nb}_{3} \mathrm{Sn}$ or $\mathrm{NbTi}$ ribbon (for example $100 \mu \mathrm{m}$ thick, 1 to $2 \mathrm{~cm}$ wide) whether or not cladded with some normal metal for thermal stability and easy joint techniques. ${ }^{112,117,129,131}$

Switches. Apart from the transformer, the switches determine the extreme pump characteristics. The switches have to carry currents in the kA range in the 'on' state and still have sufficient normal resistance $R$ in the 'off' state, so that ohmic losses remain acceptable as a condition for high efficiency. A flux pump which operates in the resistive commutation mode dissipates its commutation losses in the resistive switch which makes special high demands upon the thermostability of the switch. Inductive commutation reduces these requirements substantially.

Spatial configuration. Because the cryogenic volume should be reduced to a minimum, a compact arrangement is preferable.

Flux pump components like the transformer, switches and load coil may cause magnetic stray fields with undesirable mutual magnetic coupling. This can harmfully influence the behaviour of the superconductors and diminish the current capacity of the components. The spatial configuration has to be chosen so that these effects are minimized.

Firstly, this means that the different coils must be positioned so that the centre lines are mutually perpendicular. If necessary, application of extra aids like magnetic shields can be advantageous. Every serial selfinductance between the transformer secondary and the load coil works harmfully. The area between the secondary conductors introduces a selfinductance which has to be avoided by means of a good choice of the spatial position of the conductors.

The enormous forces between conductors in a kA fluxpump requires fastenings of sufficient mechanical strength especially if insufficiently strong conductors like certain tapes and foils are used.
Circuit joints. Superconducting plasters do not exist, so an unavoidable normal resistance due to the joints in the secondary circuit causes an extra loss and decreases the pump current with a decay time $\tau=L_{\text {load }} / R_{\text {joints }}$.

Periodical pump action can compensate for this current decay. However, it is better to reduce these losses as much as possible by minimizing the number of joints and optimizing the necessary joints. In both magnetically and thermally switched flux pumps, hard and soft superconductors in many designs occur such as $\mathrm{NbTi}, \mathrm{Nb}_{3} \mathrm{Sn}, \mathrm{NbZr}$ single core or multifilament wires in copper or copper-nickel matrix; copper or tin cladded $\mathrm{Nb}_{3}$ Sn tapes and $\mathrm{Nb}, \mathrm{NbZr}, \mathrm{NbTi}$ foils. There are very few systematic studies of joint techniques for these superconductors.

The information from this literature is very fragmentary. Every laboratory has its own unpublished 'cook-book'. Only a few flux pump papers give any information. Our research group has made a literature study on this subject and the results will be published separately. A good joint is mechanically stable, has minimum volume, a normal resistance of $10^{-10} \Omega$ down to $10^{-14} \Omega$ for the best high current joint, and causes no decrease of critical current.

Results. It may be seen from the number of references for each rectifier, that at present the half, and especially the full wave rectifier are preferred.

Some typical results are collected in Table 1 . The best results have the full wave rectifier operating in the inductive commutation mode eventually with saturation reactors. $8 \mathrm{kA}$ is the highest current reached until now. Further development focuses on the flux pump switches which have until now set a limit to the maximum current.

\section{Flux pump characteristics}

In this section, a general review of the theory of both dynamos and rectifiers is given. Qualitative conclusions concerning the efficiency in the two transfer modes are deduced.

The equations and diagrams describing their behaviour are collected in Tables 2 and 3, respectively.

Inspection of Table 2 shows explicitly the theoretical conformity of the devices. Qualitatively, the only essential point is whether or not an inductive commutation is used. Therefore the diagrams of Table 3 show only the difference between these two possible modes of operation in the case of the transformer rectifiers. In the case of dynamos, only the resistive transfer has been of interest.

Load voltage. For the homopolar dynamo flux pump, the load voltage per pole consists of three terms and is described and accepted by several authors. ${ }^{49,51,54,60,62}$

$$
\bar{V}_{\mathrm{L}}=f \phi_{0}-2 f I I_{\mathrm{L}}-f^{2} \phi_{0} \frac{l}{R},(l \ll L)
$$

$\bar{V}_{\mathrm{L}}$ is the average of the load voltage taken over one cycle. $2 l$ and $R$ are the effective selfinductance and resistance respectively, of the normal region and may depend among other variables on the velocity. ${ }^{82,84}$

The low frequency approximation in which the values of $l$ and $R$ are assumed to be constant is given here. For $n$ poles $\bar{V}_{\mathrm{L}}$ is $n$ times greater. In the case of rectifiers, the calculations 
Table 1. Some results of transformer rectifier flux pumps

\begin{tabular}{|c|c|c|c|c|c|c|c|c|c|}
\hline Author reference & Type figure & Switch & $\operatorname{lmax}_{\text {time }} A$ & $E_{\mathrm{L},} \mathrm{J}$ & $n, \%$ & $f, \mathrm{~Hz}$ & $L_{\text {load, }} H$ & $p_{\mathrm{L}}, W$ & Input transformer \\
\hline Homer $^{123}$ & $\begin{array}{l}\text { Full wave, } 17 \\
\text { Inductive transfer }\end{array}$ & $\begin{array}{l}\text { NbTi wire } \\
\text { Thermal }\end{array}$ & 1200 & 6480 & 93 & 10 & $9 \mathrm{~m}$ & $<38$ & $\begin{array}{l}8 \mathrm{~A} / 90 \mathrm{~V} \\
1320: 2 \\
\text { air core solenoid }\end{array}$ \\
\hline Bernat $^{125}$ & Full wave, 17 & NbTi wire & $\begin{array}{c}650 \\
16 \mathrm{~min}\end{array}$ & 1690 & & 0.2 & $8 m$ & & $\begin{array}{l}600: 3 \\
\text { air core solenoid }\end{array}$ \\
\hline Atherton ${ }^{129}$ & $\begin{array}{l}\text { Full wave, } 17 \\
\text { Inductive transfer }\end{array}$ & $\begin{array}{l}\mathrm{Pb} \text { foil } \\
240 \mu \Omega, \text { magnetic } \\
\mathrm{ac}+\mathrm{dc}\end{array}$ & $\begin{array}{l}275-425 \\
300 \mathrm{~S}\end{array}$ & $<16$ & 75 & $3-10$ & $180 \mu \mathrm{m}$ & $16 \mathrm{~m}$ & $\begin{array}{l}1544: 2 \\
2 A \text {, air core } \\
\text { solenoid }\end{array}$ \\
\hline Atherton ${ }^{132}$ & $\begin{array}{l}\text { Full wave, } 17 \\
\text { Inductive transfer }\end{array}$ & $\begin{array}{l}\mathrm{Nb}_{3} \mathrm{Sn} \text { ribbon } \\
\text { Thermal } \\
350-500 \mu \Omega\end{array}$ & $\begin{array}{l}1000 \\
350 \mathrm{~s}\end{array}$ & $<90$ & $<55$ & $0.8-2$ & $180 \mu \mathrm{m}$ & 0.5 & $\begin{array}{l}1544: 2 \\
6.8 \mathrm{~A} \text { air core } \\
\text { solenoid }\end{array}$ \\
\hline Droege $^{124}$ & $\begin{array}{l}\text { Full wave, } 17 \\
\text { Inductive transfer }\end{array}$ & $\begin{array}{l}\text { NbTi wire } \\
\text { Thermal } \\
0.15 \Omega\end{array}$ & $\begin{array}{l}100 \\
50 \mathrm{~min}\end{array}$ & 5000 & 87 & 0.04 & 1 & & $\begin{array}{l}40: 1 \\
25 \mathrm{~A} / 24 \mathrm{~V} \\
\text { air core solenoid }\end{array}$ \\
\hline Britton $^{117}$ & $\begin{array}{l}\text { Full wave, } 17 \\
\text { Resistive transfer }\end{array}$ & $\begin{array}{l}\text { Pb alloy } \\
\text { Magnetic ac + dc }\end{array}$ & 700 & & 71 & 60 & & 3 & $\begin{array}{l}250: 4 \\
\text { air core solenoid }\end{array}$ \\
\hline Buchhold $^{102}$ & $\begin{array}{l}\text { Full wave, } 17 \\
\text { Saturation } \\
\text { reactors }\end{array}$ & Nb tape & $500-1000$ & $<225$ & \pm 94 & $5-10$ & & $3-18$ & \\
\hline Boom $^{112}$ & $\begin{array}{l}\text { Full wave, } 17 \\
\text { Inductive transfer }\end{array}$ & $\begin{array}{l}\mathrm{Nb} 1 \% \mathrm{Zr} \text { tape } \\
10^{-4} \Omega \text { magnetic }\end{array}$ & 8000 & 2650 & $80-97$ & 0.5 & $80 \mu \mathrm{m}$ & 50 & $10 \mathrm{~A} / 20 \mathrm{~V}$ \\
\hline Dronov $^{131}$ & $\begin{array}{l}\text { Half wave, } 16 \\
\text { Resistive transfer }\end{array}$ & $\begin{array}{l}\mathrm{Pb} \mathrm{3.8 \%} \mathrm{Sn} \\
5.5 \mathrm{~m} \Omega \\
\text { magnetic }\end{array}$ & $\begin{array}{l}100 \\
15 \mathrm{~min}\end{array}$ & 0.75 & & 1.5 & $150 \mu \mathrm{m}$ & & $\begin{array}{l}800: 1 \\
\text { air core solenoid }\end{array}$ \\
\hline Fasel $^{118}$ & $\begin{array}{l}\text { Full wave } \\
\text { bridge } 18 \\
\text { Resistive transfer }\end{array}$ & $\begin{array}{l}\text { cryodiode } \\
\text { magnetic } \\
\mathrm{Pb} 1 \% \mathrm{Bi}\end{array}$ & $\begin{array}{l}6.8 \\
35 \mathrm{~min}\end{array}$ & 95 & 27 & 6 & 4.1 & & $\begin{array}{l}1000: 10 \\
\text { iron core solenoid }\end{array}$ \\
\hline Laquer $^{99}$ & $\begin{array}{l}\text { Half wave } \\
\text { automatic, } 21 \\
\text { Resistive transfer }\end{array}$ & $\begin{array}{l}\text { Switching coil } \\
\mathrm{Pb} \text { or } \mathrm{PbSn}\end{array}$ & $\begin{array}{l}34-184 \\
15-20 \text { min }\end{array}$ & & & $0.2-0.02$ & & $0-300$ & $n 500: 5$ \\
\hline Laquer $^{91}$ & $\begin{array}{l}\text { Half wave, } 16 \\
\text { Resistive transfer }\end{array}$ & $\begin{array}{l}\text { Thermal } \\
\mathrm{Nb}\end{array}$ & 1070 & 10 & & & $18 \mu \mathrm{m}$ & & iron core \\
\hline
\end{tabular}

are straightforward and done by an analysis of the rectifier network with $l, R$ and $L$ in the configurations described in the previous paper. $9,128,131,156$

In the schemes, $l$ is the selfinductance of the whole (half wave rectifier) of the secondary branch. The selfinductance additional to the transformer, and the load coil due to the leads and the switches has to be minimal but are included in $l$. We have confined ourselves to the half wave and full wave rectifiers of Figs 16 and 17 (part 1). Other schemes will give comparable results. The derivations are made assuming a trapezoidal primary wave form (Fig. 26a) where $\delta I$ is zero for the resistive transfer mode. $\delta$ is the fraction of half the period which is used for pumping, so $\delta=2 f \Delta t_{3}$.

In the resistive commutation mode, the load voltage $V_{\mathrm{L}}$ is confined to two terms; assuming that $(f l / R)$ is negligable (of the order of $10^{-4}$ ) compared to 1 and $l \ll L$ (Table 2). The first one represents the secondary induced positive transformer voltage; the second one represents the voltage drop due to switching losses. For the inductive commutation mode, this last term disappears and the first one becomes time dependent $\bar{V}_{\mathrm{L}}=f \phi(t)=f M I_{\mathrm{p}}(t)=f M\left[2 I_{\mathrm{p}}-\delta I(t)\right]$ assuming that the total primary amplitude remains constant. 


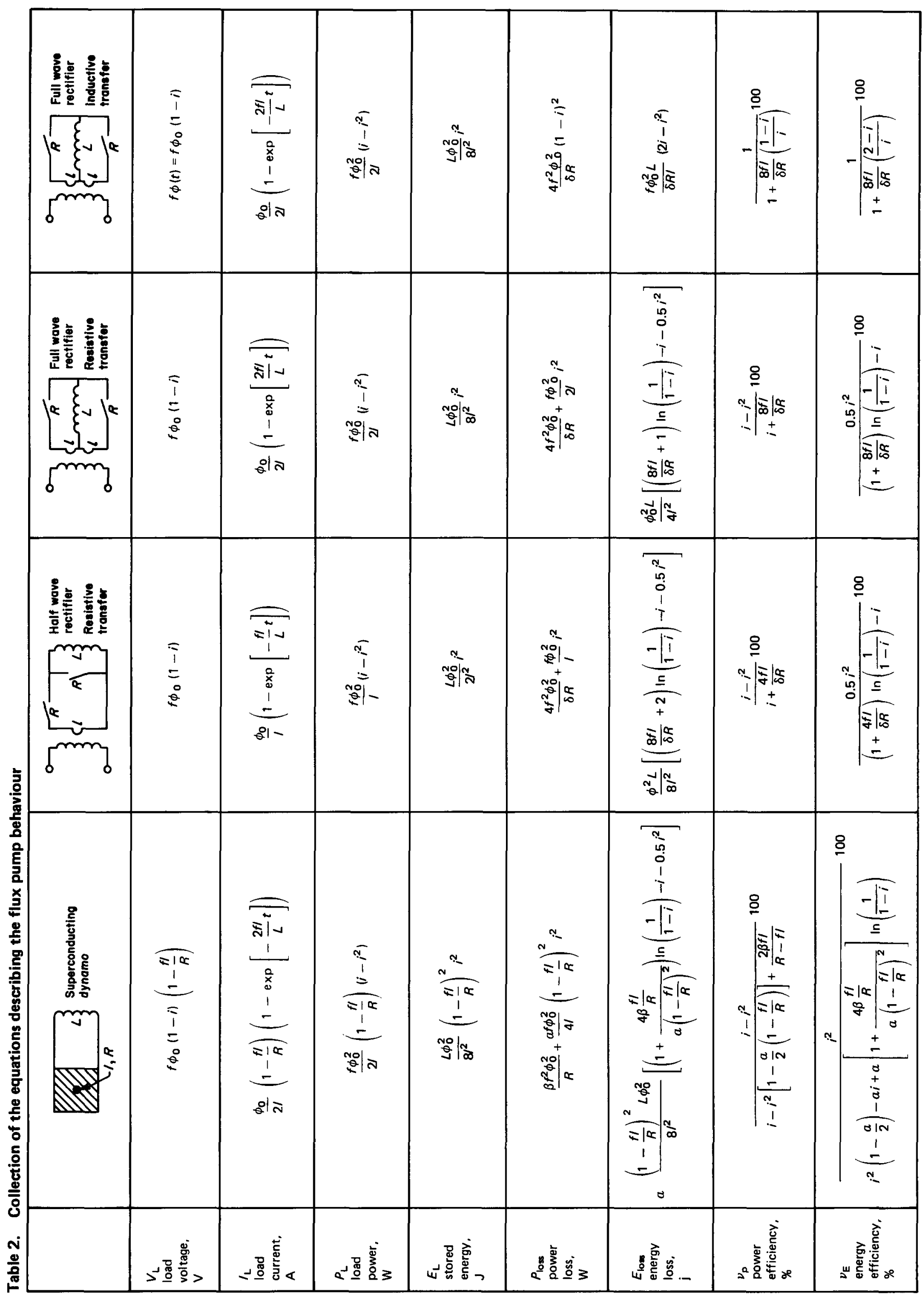


$\delta I(t)$ is the load current and so the time dependent commutation step (Fig. 26). For both modes the load voltage is the same.

The maximum load current is found for $\bar{V}_{\mathrm{L}}=0$. Substitution of $I_{\mathrm{L}}=I_{\max } i$ in the load voltage gives the equation of Table 2 .

Load current. The load current follows from the differential equation $V_{\mathrm{L}}=L \dot{I}_{\mathrm{L}}$ and has the expected form due to a saturation effect.

The maximum load current is abtained for $\bar{V}_{\mathrm{L}}=0$ or $\dot{I}_{\mathrm{L}}=0$. This maximum load current is the current that can be induced in the shortcircuited (both switches closed) transformer secondary.

The load current curve can also be obtained from a flux conservation consideration. Rewriting $I_{\mathrm{L}}$ as given in Table 2 gives the charging time $\tau$

$$
\tau(i)=\frac{1}{N} \frac{L}{f l} \ln \left(\frac{1}{1-i}\right)
$$

where $n$ is the number of poles or secondary branches. At maximum power and half the maximum current:

$$
\tau(1 / 2)=\frac{1}{N} 0.69 \frac{L}{f l}
$$

The charging time in case of the half wave rectifier is doubled compared with that of the full wave rectifier because now half of the period is effectively used for pumping.

Load power $\left(P_{L}\right)$ and stored energy $\left(E_{L}\right)$. The load power is given by $P_{\mathrm{L}}(i)=\bar{V}_{\mathrm{L}} I_{\mathrm{L}}$ and has a parabolic shape with a maximum at half the maximum current $(i=1 / 2)$ for all the considered devices, which is quite remarkable.

The stored energy equals $1 / 2 L I_{\mathrm{L}}^{2}$

Losses of power ( $P_{\text {Losd }}$ ) and energy ( $E_{\text {Loss }}$. Several authors give expressions for the dynamo losses per pole

$$
P_{\mathrm{Loss}}=\alpha f I_{\mathrm{L}}^{2}+\beta \frac{f^{2} \phi_{0}^{2}}{R}
$$

in which $\alpha$ and $\beta$ are geometrical factors determined by the ratios of normal spot, pump bridge sizes and the going patch of the magnet..$^{51,60,62,79}$ In the case of rectifiers the power loss is given by

$$
P_{\text {Loss }}(i)=\frac{\bar{V}_{R}^{2}}{R},
$$

in which $V_{\mathrm{R}}$ is the voltage drop over the open switch with resistance $R$, the complete secondary transformer voltage. In the resistive commutation mode two terms arise: a switching loss term linear with $I_{\mathrm{L}}^{2}$ and an ohmic loss term. When the condition $f l / R \ll 1$ is not fulfilled, an extra loss term linear with $I_{\mathrm{L}} \exp (-R / f l)$ cannot be neglected. In the inductive commutation mode the switching loss term disappears.

Because the effective primary pump amplitude decreases linearly with $I_{\mathrm{L}}$ in this mode, also the ohmic losses decrease until zero for $i=1$.

The energy losses become $E_{\text {Loss }}=\int_{0}^{\tau} P_{\text {Loss }} \mathrm{d} t$ and are included in Table 2.

Power and energy efficiency are defined as:

$$
\eta_{\mathrm{p}}=\frac{P_{\mathrm{L}}}{P_{\mathrm{L}}+P_{\mathrm{Loss}}} 100 \%, \quad \eta_{\mathrm{E}}=\frac{E_{\mathrm{L}}}{E_{\mathrm{L}}+E_{\mathrm{Loss}}} 100 \%
$$

and are referred to the cryogenic environment only.
Moreover, only the efficiency of the rectifying process is treated and not the efficiency of the complete flux pump. The additional losses like ac losses and heat switch losses, can sometimes be substantial. One has to be on the alert for the indifferent use of both energy and power efficiency. The energy efficiency seems to be the best and most honest criterion for evaluation. In the literature both concepts are used.

In Table 3, for example, the full wave rectifier case is treated with the value of $(8 f l / \delta R)$ as a parameter.

The diagrams show four curves whereby the value of $(f l / R)$ changes by a factor of 10 from curve to curve.

The maxima of the power efficiency lie on the line $\eta_{\mathrm{p}}=\left(1-2 i_{\max }\right) 100 \%$ in the resistive transfer mode. The maxima of the energy efficiency lie approximately on the line $\eta_{\mathrm{E}}=\left(1-1.41 i_{\max }\right) 100 \%$.

In the inductive transfer mode the maxima appear for $i=1$ in both modes. It is seen from the diagrams that the difference between resistive and inductive commutation mode is marginal if a flux pump works until $i$ is small, this being a pump with an overlarge capacity.

However, maximum power appears at $i=1 / 2$. So that it is very attractive to use the flux pump until $i=1 / 2$ or more. In this case the behaviour of the losses and the efficiency is for the inductive commutation mode which are different from and essentially better than those in the transfer mode. ${ }^{9,128}$

The most important flux pump parameter is obviously $(f l / R)$. So an ideal flux pump works at a very low frequency, has a very small $l$ and a very high or infinite $R$. With $(f l / R)$ approaching zero, an energy efficiency of almost $100 \%$ is possible. $^{7}$

It becomes very understandable now why with a transformer rectifier flux pump a much higher efficiency is possible than with a homopolar dynamo flux pump. In these 'sheet' pumps, the magnitude of $l$ and $R$ are coupled via the normal spot dimensions so that their ratio cannot be made small enough.

In the rectifier case $l$ and $R$ are principally uncoupled and much easier to manipulate. So consequently their ratio can be made very small $\left(<10^{-4}\right)$ or even zero for mechanical breakers $(R=\infty)$. If a high efficiency of the rectifying process is reached it becomes very important to reduce all extra losses which reduces the efficiency of the complete flux pump.

\section{Rectifier switches}

The ideal switch (power cryotron) for use in a flux pump has to fullfil several requirements: zero resistance and a large current carrying capacity in the 'on' -state, an infinite resistance in the 'off' -state. The latter minimizes the ohmic losses. The selfinductance of the switch has to be zero in order to attain maximum load current and to minimize the commutation losses. Also the magnetic coupling then reduces with respect to the surroundings.

Short switching times permit the use of higher flux pump frequencies and therefore the charging time of the load coil is shortened. Furthermore, accompanying losses in heaters or switch coils have to be minimal. From a cryogenic point of view, the volume has to be as small as possible.

It is evident that these requirements oppose each other or are inconsistent. For instance, the requirements of small volume, small area/volume ratio and rapid cooling are difficult to fulfil at the same time. More difficult even in a heat 
Table 3. Diagrams illustrating the flux pump behaviour

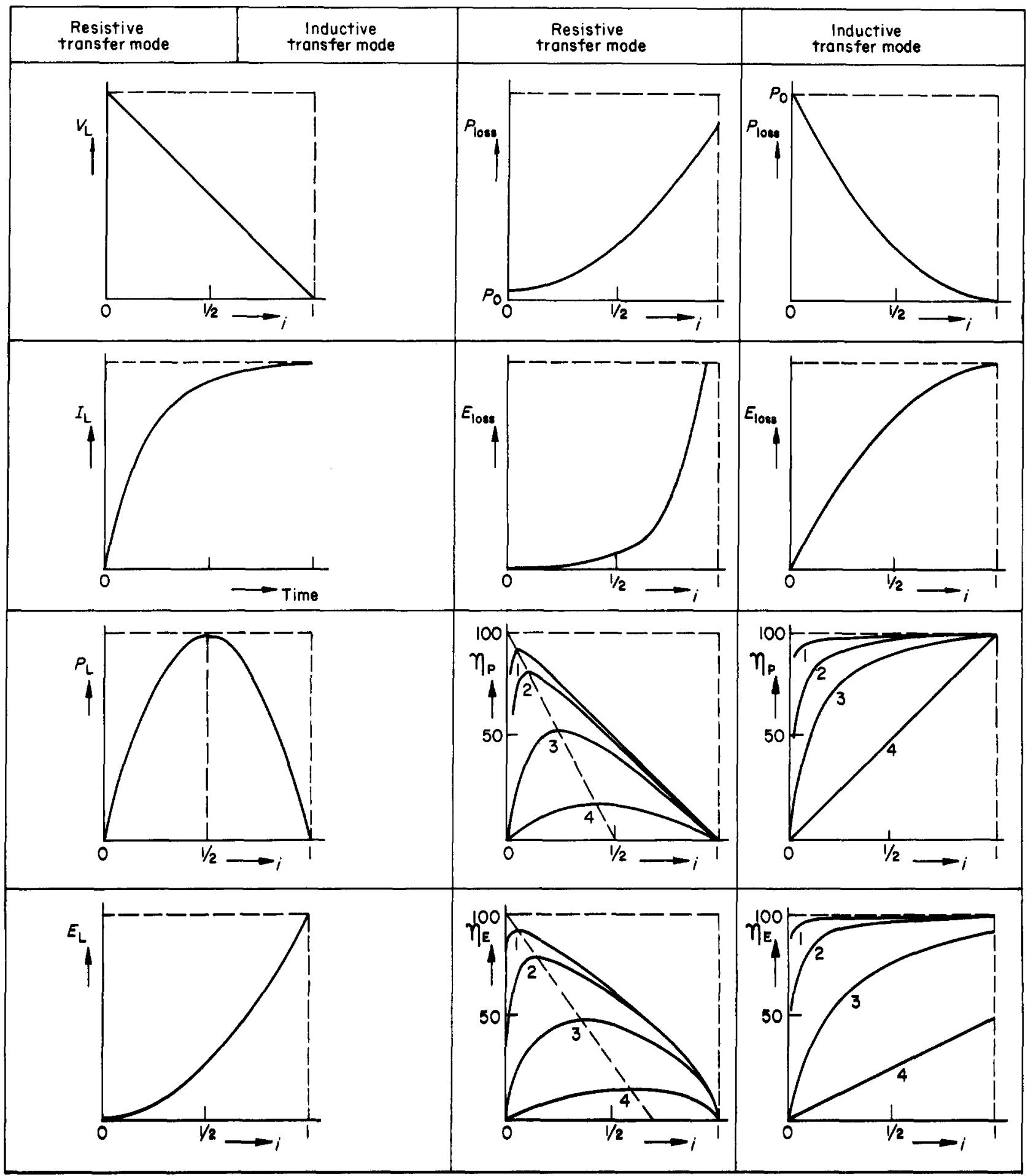

The parameter by the curves $(8 f / / \delta R)$ is $10^{-3}, 10^{-2}, 10^{-1}$ and 1 for the curves 1 to 4 respectively

driven switch are the requirements for short switching-on time, ie, good thermal insulation, and short switching-off time, ie, good thermal contact with the environment.

Several methods have been used to meet the requirements of an ideal switch. The following operation modes can be distinguished: one, magnetic switch: the superconducting to normal transition is induced by an external magnetic field. Two, thermal switch: heating and cooling the switch material induces the transition. Three, combined thermal and magnetic switch. Four, current or magnetic pulsed switch: the transition to the normal state is induced by a current or magnetic pulse, and five, mechanical breakers.
The advantages and peculiarities of these devices will be discussed below. The use of superconductors as switch material results in a zero resistance in the 'on'-state. Consequently the problem is to create sufficient resistance in the 'off'-state so that the leakage currents remain limited. It can be seen from Table 1 that both thermal and magnetic switches used in flux pumps have a normal resistance in the $\mu \Omega-\mathrm{m} \Omega$ range.

The requirements of rectifier switches are that they have to be well distinguished from the high current, high voltage protection, persistent current switches, fault current limiters etc. 
Magnetic switch. A certain volume of superconductor alternates between the normal and superconducting state in a regulated magnetic field. In practice, the ac magnetic field generated by some coil configuration will be limited to about $1 \mathrm{~T}$. Otherwise the configuration of switch coils with their selfinductance make the switch too slow and/or they need large power supplies. The allowable switch coil current is usually limited for thermal loss reasons to a low level, typically below $100 \mathrm{~A}$.

The Gorkov-Goodman relation couples the value of $\rho_{\mathrm{n}}$ and $H_{\mathrm{c}}$. The practical limit for $B_{\mathrm{c},}$ to maximal $1 \mathrm{~T}$ determines maximum values for $\rho_{\mathrm{n}}$ and $f_{\mathrm{c}}$. Thin foils, films, parallel wires or multifilament tapes can be used as conductors.

The design goal current $l_{\mathrm{L}}$ determines the conditions for the cross section of the conductor. For safety reasons one takes the cross section a factor four or five greater that needed to carry the design current. ${ }^{112,114}$

A given cross section in combination with a desired normal resistance $R$ determines the length and thus the volume $\left(V=I^{2}{ }_{\max } R / \rho_{\mathrm{n}} J_{\mathrm{c}}^{2}\right.$ ) of the switch element. When maintaining a normal resistance $R$, the problems with the conductor volume in the switch coil become considerable. With increasing width of superconductive strips, the current carrying capacity becomes constant. ${ }^{12,137}$ Placing the flat conductor between flat hard superconductors or using multifilament tapes may overcome this phenomenon. ${ }^{134,135,137}$ Otherwise for widths greater than 1 to $2 \mathrm{~cm}$, depending on the material, parallel conductors are preferable. Very thin films can be switched with a very small amplitude of a pulsed perpendicular field (large $\mathrm{d} B / \mathrm{d} t, 200 \mathrm{Ts}^{-1}$ ). With these short rise times, flux jumps are initiated. ${ }^{153}$

Type II superconductors have a less sharp transition than a type I superconductor material but a higher $\rho_{\mathrm{n}}$ and $J_{\mathrm{c}}$. Frequently used materials are $\mathrm{Pb}, \mathrm{Pb}$ alloys, $\mathrm{Nb}$ and dilute $\mathrm{Nb}$ alloys. An easy optimum $\mathrm{Pb}$ alloy $(\mathrm{Pb}, \mathrm{Zn}, \mathrm{Ln}, \mathrm{Sn}, \mathrm{Ga}, \mathrm{Hg}$ ) is being researched; a relation between $H_{\mathrm{c}}, \rho_{\mathrm{n}}$ and the melting temperature has been found. For a given $H_{\mathrm{c}}, \rho_{\mathrm{n}}$ can be increased changing the alloy mixture which, however, results in a lower melting temperature. A good compromise seems to be: $\mathrm{Pb} 50$, Sn 44 , In $5, \mathrm{Ga} 0.3 ; H_{\mathrm{c}}=17000 \mathrm{e} ; \rho_{\mathrm{n}}=4.4 \times 10^{-8} \Omega \mathrm{m}^{114,117}$ An artificial increase of $\rho_{n}$ is achieved with insulating enclosures or alternating packing of thin superconducting and insulator films. ${ }^{138} \mathrm{~A}$ consideration of the necessary switching pulse power sets an optimum $\rho_{\mathrm{n}}$ to 1 to $2 \mu \Omega^{127,152}$

The total loss in the switch determines an optimum conductor length because the ac losses in the field coil and switch element are proportional to this conductor length, while the leakage current losses are inversely proportional to the conductor length. ${ }^{79,114}$

The switch is not allowed to increase the secondary selfinductance in the flux pump. Therefore, a non inductive configuration within a minimum volume is necessary.

Many more or less non-inductive configurations are possible. ${ }^{144}$ The conductor layers may be bifilar wound (Fig. 27) or pressed into a sandwich model (Fig. 28).

The electrical insulation between the layers must allow for a good thermal conduction to the helium bath. Possible solutions are: spacers, cooling channels, porous insulators, oxidized metals such as $\mathrm{Al}$, insulating plastics etc.

The amount of heat production allowed in the magnetic switch depends on the geometry, conductor and insulation materials. Film boiling isolates the conductor from the helium bath. A heat production of 0.4 to $0.8 \mathrm{~W} \mathrm{~cm}^{-2}$ seems to be tolerable. ${ }^{118}$ In order to avoid mechanical losses and internal damage, the construction is mechanically stable because large forces act between the layers when heavy currents flow.

A switch element placed in a parallel magnetic field gives the sharpest transition. In a perpendicular field, flux penetrates the switch element earlier due to the demagnetisation effect. However, the final magnetic field required for total transition remains the same so the transition is more gradual. ${ }^{127}$ Thin foils are better stabilized in a parallel field. The field coil may be a solenoid or a toroid which avoids stray fields. The toroid can be wound around the switch element $^{114}$ or the (parallel) switch elements can be mounted in the airgaps of the toroid. ${ }^{112}$ Both rectifier switches can be combined in one coil configuration. ${ }^{122}$

Many different configurations are possible with solenoidal field coils. For example, the switch element can simply be placed in the centre of the ac coil,,$^{95,108,114}$ between two concentric series connected oppositely wound coils, or between two concentric coils where one coil supplies a dc bias field and the other one of the switching ac field..$^{117}$

The latter method is preferable with switch materials which have a relatively high current carrying capacity just below the critical field. Magnetic shields (coil or cylinder) of a hard superconductor or ferromagnetic materials may concentrate

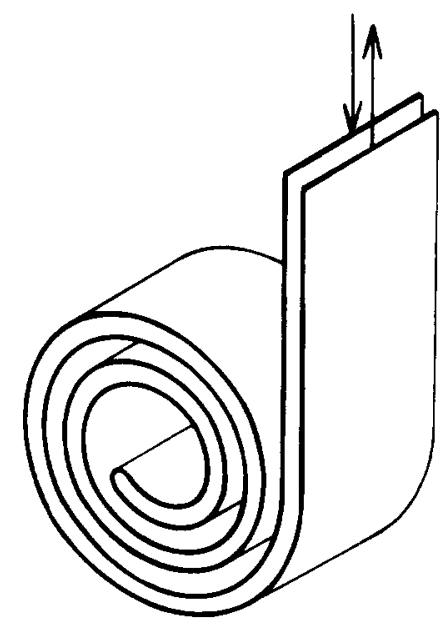

Fig. 27 Bifilar wound swiřch element

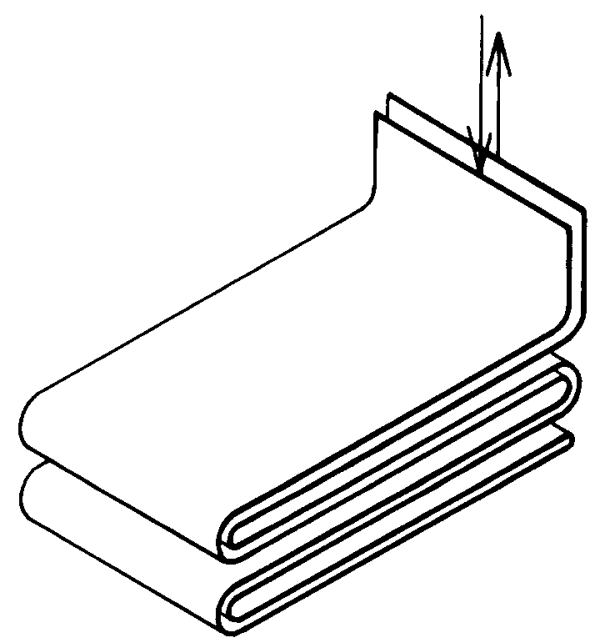

Fig. 28 Bifilar sandwich switch element 


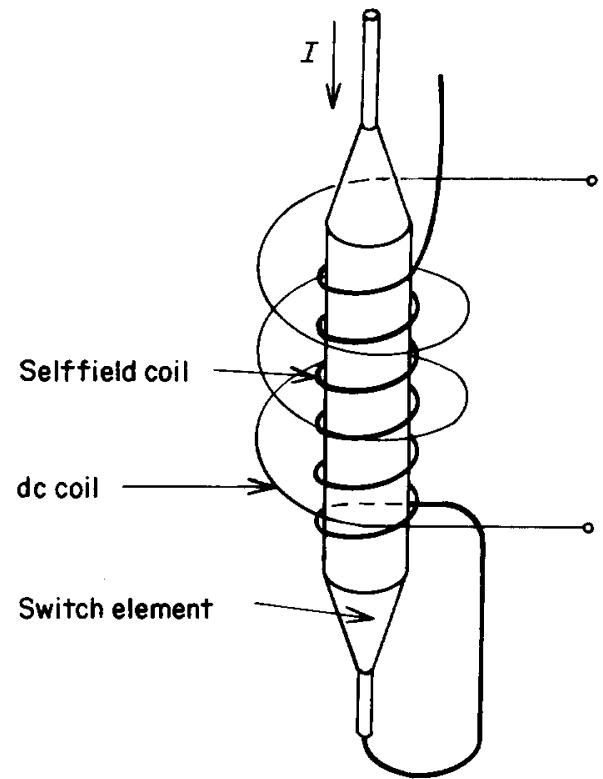

Fig. 29 Automatic self switching element, called cryodiode

the magnetic field at the switch element. Such a magnetic shield can decrease the selfinductance of the switch coil by a factor of $\operatorname{six}^{131}$ which has the advantage of more rapid switching.

An automatic self switching element, called cryodiode, is shown in Fig. 29.111,118,142

The self-magnetic field is parallel or anti-parallel to the external bias field depending on the current direction. In one direction both magnetic fields add and exceed the critical value; the switch then opens. In the opposite direction, both fields oppose each other and the resulting field remains below the critical value, the switch is now closed.

The limiting current through the cryodiode is that current which can make the cryodiode resistive even when the selfmagnetic field is anti-parallel to the bias field. By use of the full wave rectifier flux pump, the secondary current direction alternates due to the primary ac voltage making the cryodiodes alternately conducting. Such a cryodiode increases the secondary selfinductance of the flux pump and makes, compared with a standard switch, reverse operation of the flux pump impossible.

Thermal switches. In these switches, a heat source raises the temperature of the superconductor above its critical temperature. Compared with magnetic switches, the use of superconductors is not limited to those with a relatively low critical field.

Now the selection of a high $\rho_{\mathrm{n}} J_{\mathrm{c}}^{2}$ superconductor $(\mathrm{NbTi}$ : $\left.7 \times 10^{13} \mathrm{Wm}^{-3}, 10 \mathrm{~K} ; \mathrm{NbN}: 9 \times 10^{16} \mathrm{Wm}^{-3}, 16 \mathrm{~K}\right)^{154}$ allows a very compact volume of the switch, provided that the critical temperature $T_{\mathrm{c}}$ and the specific heat are suitably chosen to avoid too large thermal losses. However, there is a fundamental conflict between good thermal insulation of the switch during the resistive state and a good thermal conduction to the helium bath during the recovery state of the switch.

A theoretical analysis may give a suitable compromise for this problem. Usually the switch element consists of one or more parallel multifilament $\operatorname{NbTi}\left(T_{\mathrm{c}}=9\right.$ to $\left.10 \mathrm{~K}\right)$ wires in $\operatorname{CuNi}\left(\rho_{\mathrm{n}} \approx 35 \times 10^{-8} \Omega \mathrm{m}\right)$ or $\mathrm{Cu}\left(\rho_{\mathrm{n}} \approx 35 \times 10^{-10} \Omega \mathrm{m}\right)$ matrix. Removing the matrix material makes the conductor unstable in the zero resistance state, but allows more rapid switching and a relatively high ohmic resistance. In the case of copper, the matrix must be (partly) removed otherwise it acts as a shortcircuit of the 'normal' superconductor. In the case of a coppernickel matrix, the matrix need not be removed to have limited conductor stabilization.

Recently $\mathrm{Nb}_{3} \mathrm{Sn}$ ribbon in bronze matrix has been applied. ${ }^{132}$ Compared with $\mathrm{NbTi}$, it requires a relatively high heat power because of the high $T_{\mathrm{c}}$ of $18.3 \mathrm{~K}$, if the apparatus works at $4 \mathrm{~K}$. For higher working temperatures this argument may not be relevant. Further development on thermal $\mathrm{Nb}_{3} \mathrm{Sn}$ switches is necessary because $\mathrm{Nb}_{3} \mathrm{Sn}$ is a very feasible high current conductor essential for many commercial applications of superconductivity.

The conductor is made normal with heater wires or plates wound around or interwoven with the switch element wires. ${ }^{106,124,125,132}$ Thermal switching of the transformer secondary winding allows an elegant compact design. ${ }^{123}$

Warm helium gas switches constructed up until now have been unreliable. ${ }^{9108}$ The application of light, particles or $\mathrm{X}$-ray beams ${ }^{147}$ seems attractive but is more difficult to realize. ${ }^{139,149}$ A direct energy gap transition $\left(h \nu=2 \Delta \approx 3.5 k_{\mathrm{B}} T_{\mathrm{c}}\right)$ produces no extra heat but is not feasible because it requires a far infra red frequency $\left(\nu= \pm 70 T_{\mathrm{c}} \mathrm{GH}_{\mathrm{z}}\right)$, which lies in the transition region between practical lasers and masers.

Current-or magnetic pulsed power switches. A current pulse through a superconductor in a non inductive configuration disturbs the current carrying capacity so that the superconductor becomes normal. This trigger pulse may be generated by a condenser bank or magnetically by a switch coil. Such triggered switches are used as short-circuiting elements for storage coils and should allow a fast transducer in the milli-or microsecond range of the stored energy to an inductive load such as plasma compression coils or to a resistive load such as pulsed laser systems.

Such use of switches poses special requirements including very short rise times, good electrical insulation because of the very high induction voltages in the $\mathrm{kV}$ range and good mechanical stabilization. Generally these stabilization demands bring about great switch recovery times and a large volume. The slow recovery of even some minutes makes these switches unsuited for flux pumps. An evaluation of these designs may however be helpful for research on flux pump switches.

Mechanical breakers. In the 'off'-state the resistance of the mechanical breaker is infinite. However, the absence of sufficient low resistance in the 'on'-state of the presently known designs limits the application of mechanical breakers in flux pumps. The total contact resistance is given by the resistance due to oxide films on the contacts, the resistance of the holder and the constriction resistance, because the current is constricted at the real area. The dominant constriction resistance of a point contact is the dominant contribution. ${ }^{146,150}$

To obtain a low resistance it is advantageous to use soft, pure, normal metals or superconductors and press the contacts against each other with a large force. The contacts must be kept oxide free. They can be placed in vacuum $^{145,146,150,151}$ or be self-cleaning. ${ }^{12,140}$ Filling the switch house at room temperature with a suitable protective gas, a vacuum in the housing is created at liquid helium temperature. ${ }^{143}$ Vacuum type mechanical breakers, for use as a persistent current switch in superconducting systems 
with a maximum current of $500 \mathrm{~A}$ and a minimal resistance of $0.025 \mu \Omega$ are possible using $\mathrm{NbTiZr}$ alloy in a $\mathrm{Cu}$-matrix for stability. ${ }^{145}$ Plating the contacts with thin films ${ }^{133,136,143,155}$ may lower the resistance. Best results are reported with Ag-films of about $10 \mu \mathrm{m}$ thickness. ${ }^{133}$ The contacts can also be hatched with grooves ${ }^{145}$ making a good contact when strongly pressed. ${ }^{141}$ Superconducting sliding contacts have been made, but only in the low current range. ${ }^{148}$

\section{Evaluation}

A flux pump is a potentially attractive apparatus for many cryogenic applications where a high current within a relatively small selfinductance is required.

In the beginning the so called flux compressors were of interest but further development was stopped because of their failures which prevented them from general use. The dynamo flux pumps have reached currents up to $15 \mathrm{kA}$ and are being designed for $50 \mathrm{kA}$. The technique is simple, but the theoretical understanding is insufficient. Efficiencies above $60 \%$ are not practically possible with such dynamos. Therefore their use is limited to low power but high current applications such as in test beds. Both flux compressor and dynamo are generally mechanically driven.

The superconducting rectifier and a fully electrical flux pump makes nearly $100 \%$ efficiency possible during the whole pump process if it operates in the inductive commutation mode. The theoretical behaviour of such devices is well understood. The highest current possible until now has been $8 \mathrm{kA}$. The main obstacle for better results is the construction of the high current switches which have to operate in the frequency range from 0.1 up to $50 \mathrm{~Hz}$.

In principle transmission of high powers is feasible with rectifiers due to the high efficiency of the conversion, but the required high voltages resulting from higher frequencies will oppose the requirements for high efficiency. So there seems to be a fundamental conflict between high power and high efficiency. Nevertheless, for special purposes dynamos and recitifiers can offer suitable and even sometimes economical solutions.

Until now flux pumps have had a power in the range from $1-500 \mathrm{~W}$ and have charged relatively small coils with selfinductances from $10 \mu \mathrm{H}$ to $10 \mathrm{mH}$ and a stored energy less than $10 \mathrm{~kJ}$. In case of larger selfinductances of the load coils the large charging time becomes a serious problem owing to the low voltages. The charging time can be reduced by over-sizing the capacity (small $i$ ), and by allowing a higher frequency (lower efficiency). Also a greater input flux $\phi_{0}$ will reduce the load time.

Today there is only an incidental use of flux pumps in spite of the general conviction that they could play an important role for many purposes. Even a low efficiency flux pump from the point of view of energy cost is more attractive than a conventional power supply for currents above some hundred amperes. ${ }^{10}$

In conclusion it can be said that the investigations carried out so far only have a relatively small connection with real applications.

Some applications which have been proposed are: a $1 \mathrm{kA}$ loss make up device (rectifier) for the superconducting magnets for the Canadian Magler vehicle. ${ }^{122,126,128,129,132}$ a $5 \mathrm{kA}$ rectifier for superconducting particle accelerator magnets; ${ }^{\mathbf{1 2 0}}$ a 250-700 A rectifier for beam splitter and beam bending magnets, ${ }^{117}$ a $1 \mathrm{kA}$ dynamo for energizing the field windings of a superconducting generator ${ }^{80,82}$ a $50 \mathrm{kA}$ dynamo for a superconducting transmission line test bed. ${ }^{77}$

Further development is needed on the following issues: new materials and conductors for use in magnetic switches and transformers with low losses under ac 1-50 Hz conditions; optimum switch coil geometries in the case of magnetic switches; construction of a high current ac conductor which is optimized for use in thermal switches, reduced stability versus a suitable resistivity above the critical temperature; joint techniques for different types of conductors in the various parts of the rectifier secondary circuit.

The relation between the use of a flux pump in a large magnet system and the general protection of that system has so far been insufficiently studied. Its solution is essential.

The authors are indebted to Mr G. Tiemessen for his study of superconducting switches and joint techniques and to Prnfessor L.C. van der Marel for stimulating this work.

\section{References}

$1-132$ are references in part I

\section{Switches}

133 Zar, J.L. Cryogenic switch, US patent 3349209 (1967)

134 Buchhold, T. Power cryotron, US patent 3310767 (1967)

135 Buchhold, T. Thin wire power cryotrons, US patent 3394335 (1968)

136 Atherton, D. Mechanical semi-persistent switches for superconducting solenoids, Cryogenics 8 (1968) 248

137 Newhouse, V.L. Superconducting devices, Superconductivity vol. 11, ed R.D. Parks, Marcel Dekker N.Y. (1969) 1308

138 Dietrich, I. Power-current cryotron, US patent 3488617 (1970)

139 Testardi, L.R. Destruction of superconductivity by laser light, Phys Rev B4 (1971) 2189

140 Siegwarth, J.D., Sullivan, D.B. A mechanical superconducting switch for low temperature instrumentation, Rev Sci Instrum 43 (1972) 153

141 Winter, J.J., Breslin, J.T., Gaule, G.K. High-current superconductive pressure contact studies, $J$ Appl Phys 43 (1972) 1226

142 Fasel, R. Das verhalten der Kryodiode in supraleitenden gleichrichteranlagen mit induktiver Last, Helv Phys Act, 45 (1972) 63

143 Kruger, P. Solenoid switch, particularly cryogenic switch, US patent 3715694 (1973)

144 Croso, H., Prost, G., Quefellec, A., Solé, J. US patent 3715703 (1973)

145 Jchikawa, H., Ogiwara, H. Design considerations for superconducting magnets as maglev pad, ICEC 5 (1974) 86

146 Kuwabara, K., Sugawara, H. Development of vacuum-type persistent current switches, ICEC 5 (1974) 409

147 Schmieder, R.W. Superconducting switches using radiation induced quenching, IEEE MAG-11 (1975) 590

148 Blackford, B.L., Purcell, C.J., Stroink, G. A sliding superconducting contact, Cryogenics 15 (1975) 283

149 Wolff, A.A. Laser activated superconducting switch, US patent 3956727 (1976)

150 Kuwabara, K., Sugawara, H., Miyashita, T. Persistent current switch including electrodes forming parallel conductive and superconductive paths, US patent 4021633 (1977)

151 Marsing, H., Parsch, C.P., Franksen, H. Shorting contacts for closing a superconducting current path operated by a bellows arrangement responsive to the pressure of a cryogenic medium used in cooling the contacts US patent 4024363 (1977)

152 Gray, K.E. Evaluation of superconductors for large scale switching of electrical power, IEEE MAG-13 (1977) 784

153 Gray, K.E., Lenihan, T., Tarczon, J. Thin film superconducting switches, IEEE MAG-15 (1979) 175

154 Ulbricht, A. Test results of a resistive sc-power switch of $40 \mathrm{MW}$ switching power at a voltage of $47 \mathrm{kV}$, IEEE MAG-15 (1979) 172

155 Morpurgo, M. A mechanical superconducting switch for large magnets, ICEC 8 Genova, Italy (1980) 844

156 Van de Klundert, L.J.M., ten Kate, H.H.J. Cryogenics, 214 (1981) 195-206 